\title{
Does Th1/Th2 cell imbalance affect immunoglobulin A nephropathy?
}

\author{
Tae Ryom Oh, Eun Hui Bae \\ Department of Internal Medicine, Chonnam National University Medical School, Gwangju, Republic of Korea
}

\section{See Article on Page 69-76}

Immunoglobulin A (IgA) nephropathy is the most common form of glomerulonephritis both in Korea and worldwide. Clinical manifestations of IgA nephropathy vary among patients ranging from asymptomatic hematuria to nephrotic syndrome. The renal survival rate of IgA nephropathy is poor. IgA nephropathy is characterized by mesangial deposition of immune complexes, including under-glycosylated IgA, with or without IgG and complement [1]. Despite many previous studies conducted to determine the pathogenesis of IgA nephropathy, its pathogenesis has not yet been clearly identified. Previous studies have demonstrated that IgA nephropathy is a systemic disease that is not kidney-specific $[2,3]$. Recently, functional abnormalities in T lymphocytes was suggested to be a cause of mesangial deposition of IgA with altered glycosylation, and several studies have shown that $\mathrm{T}$ lymphocytes play a major role in the pathogenesis of IgA nephropathy.

$\mathrm{CD} 4^{+} \mathrm{T}$ cells are classified by transcription factor expression; T-bet in Th1 cells, GATA3 in Th2 cells, RORyt in Th17, Foxp3 in Treg, and Bcl6 in follicular helper T cells. Each T cell subtype expresses a cell-specific cytokine and plays a unique immunological role. Thl lymphocytes contribute to cellular immunity via secretion of interleukin (IL)-2, interferon gamma (IFN- $\gamma$ ), and tumor necrosis factor alpha. Th2 lymphocytes induce humoral immunity by secreting IL4, IL-5, and IL-13 [4]. Th1 and Th2 lymphocytes modulate systemic immune system function through mutual regulation (Fig. 1). Several studies have shown a role for Th1/Th2 lymphocyte polarization and associated cytokine production in the pathogenesis of IgA nephropathy. Thl polarization was demonstrated in the early stages of IgA nephropathy in a ddY murine model of IgA nephropathy [5]. In addition, administration of Thl cytokines can result in crescentic lesions of glomeruli in hyper-IgA mice [6]. By contrast, another study showed polarization toward the Th2 response in tonsil mononuclear cells displaying IgA nephropathy [7]. To date, the pathogenic role of Th1/Th2 polarization remains unclear.

Han et al. [8] reported elevated serum IFN- $\gamma$, a Th1 cytokine, and urinary monocyte chemoattractant peptide (MCP)-1 in IgA nephropathy in comparison to healthy controls. IFN- $\gamma$ acts as a major pathogenic cytokine in IgA nephropathy, and increased IFN- $\gamma$ promotes urinary MCP1 production. Notably, this study simultaneously obtained cytokine profiles from various sources, including serum, urine, kidney, peripheral blood mononuclear cells, and

Received: February 13, 2021; Accepted: February 13, 2021

Editor: Tae-Hyun Yoo, Yonsei University, Seoul, Republic of Korea

Correspondence: Eun Hui Bae

Department of Internal Medicine, Chonnam National University Medical School, 42 Jebong-ro, Dong-gu, Gwangju 61469, Republic of Korea. E-mail: baedak@chonnam.ac.kr,baedak@hanmail.net

ORCID: https://orcid.org/0000-0002-1252-9679

Copyright @ 2021 The Korean Society of Nephrology

(a) This is an Open Access article distributed under the terms of the Creative Commons Attribution Non-Commercial and No Derivatives License (http:// creativecommons.org/licenses/by-nc-nd/4.0/) which permits unrestricted non-commercial use, distribution of the material without any modifications, and reproduction in any medium, provided the original works properly cited. 


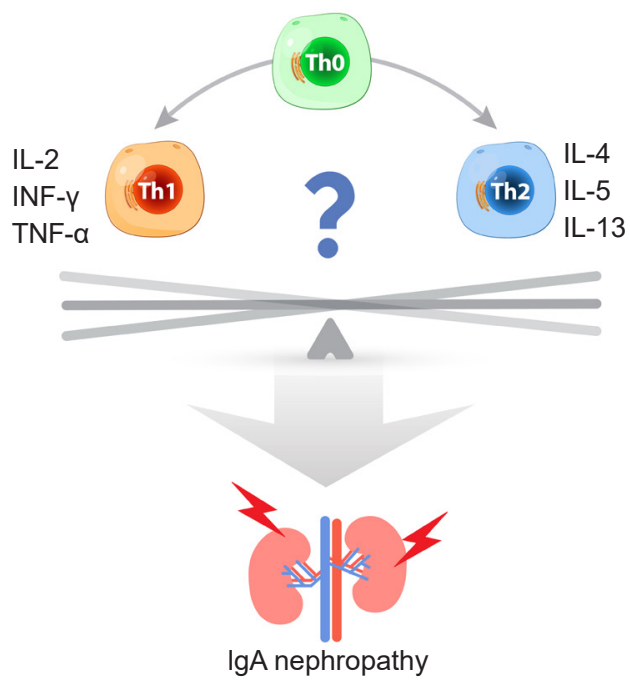

Figure 1. Polarization of Th1/Th2 cell and immunoglobulin A (IgA) nephropathy.

IL, interleukin; IFN, interferon; TNF, tumor necrosis factor.

evaluated the response of mesangial cells to Th1/Th2 cytokines. IgA nephropathy-specific Th1/Th2 polarization was also assessed. This study has important clinical significance in that they present the results of comprehensive analyses of human and in vitro experimental data, but it also has some limitations. First, similar to most studies in this field, the sample size was small. Second, temporal changes in Th1/Th2 lymphocytes and relevant cytokines were neither considered nor analyzed. Third, the study design cannot infer causal relationships. The correlation analyses provide interesting results, but they do not prove causality. Further research is needed to determine causality. These studies present clinically important results despite these limitations.

Previous studies have investigated the influence of $\mathrm{T}$ lymphocytes on the pathogenesis of IgA nephropathy. The most influential limitations of the previous studies are the small numbers of subjects and their methodology that cannot infer a causal relationship. Studies aimed at identifying specific $\mathrm{T}$ lymphocytes and relevant cytokines as therapeutic targets, or as surrogate markers for evaluating therapeutic response, have recently been highlighted, but in these studies, specific $\mathrm{T}$ lymphocytes and related cytokines were not clearly identified. Potential therapeutic strategies for treating early-stage IgA nephropathy using immunity-related $\mathrm{T}$ lymphocytes have provided accumulating evidence in support of clinical application. Much research in numerous fields must be completed to allow clinical application of new treatments utilizing $\mathrm{T}$ cell immunity. For disease monitoring and risk stratification in IgA nephropathy, further studies are required to clarify the association between renal function deterioration, histological damage, and $\mathrm{T}$ lymphocytes and their secreted cytokines. In addition, to date, no temporal analyses of $\mathrm{T}$ cell immunity over the course of disease progression have been conducted, thus studies of the efficacy of serial T cell monitoring are needed.

Well-designed large-scale future studies should elucidate the influence of Th1/Th2 polarization and related cytokines on IgA nephropathy, and these studies should facilitate the development of new therapeutic strategies that involve the regulation of related pathways. It may also be possible to control disease progression through the regulation of immunity.

\section{Conflicts of interest}

Eun Hui Bae is an Associate Editor of Kidney Research and Clinical Practice. All authors have no other conflicts of interest to declare.

\section{Funding}

This research was supported by Chonnam National University Hospital Biomedical Research Institute Grant (BCRI 20076). The sponsor had no role in study design, data collection and analysis, decision to publish, or preparation of the manuscript.

\section{Authors' contributions}

Conceptualization: TRO, EHB

Data curation: TRO, EHB

Formal analysis: TRO, EHB

Funding acquisition: EHB

Investigation: TRO, EHB

Methodology: TRO

Project administration: EHB

Visualization: TRO

Writing-original draft: TRO

Writing-review \& editing: EHB

All authors read and approved the final manuscript.

\section{ORCID}

Tae Ryom Oh, https://orcid.org/0000-0002-3713-0939 
Eun Hui Bae, https://orcid.org/0000-0003-1727-2822

\section{References}

1. Wyatt RJ, Julian BA. IgA nephropathy. N Engl J Med 2013;368:24022414.

2. Floege J. Recurrent IgA nephropathy after renal transplantation. Semin Nephrol 2004;24:287-291.

3. Hurtado A, Johnson RJ. Hygiene hypothesis and prevalence of glomerulonephritis. Kidney Int Suppl 2005;(97):S62-S67.

4. Tang Y, He H, Hu P, Xu X. T lymphocytes in IgA nephropathy. Exp Ther Med 2020;20:186-194.
5. Suzuki H, Suzuki Y. Murine models of human IgA nephropathy. Semin Nephrol 2018;38:513-520.

6. Suzuki H, Suzuki Y, Aizawa M, et al. Th1 polarization in murine IgA nephropathy directed by bone marrow-derived cells. Kidney Int 2007;72:319-327.

7. He L, Peng Y, Liu H, et al. Th1/Th2 polarization in tonsillar lymphocyte form patients with IgA nephropathy. Ren Fail 2014; 36:407-412.

8. Han SY, Jeong KH, Ihm CG, Kang YS, Cha DR. Serum interferon- $\gamma$ and urinary monocyte chemoattractant peptide- 1 are important factors in the pathogenesis of immunoglobulin A nephropathy. Kidney Res Clin Pract 2021;40:69-76. 